\title{
Nuclear Data for New Fuel Cycles
}

\author{
Bernard Haas \\ Centre d'Etudes Nucléaires in Bordeaux Gradignan, \\ CNRS/IN2P3-Université Bordeaux 1, F33175 Gradignan Cedex, France
}

Received on 26 September, 2003

\begin{abstract}
In the framework of studies concerning new fuel cycles and nuclear wastes produced by reactors, we have undertaken a number of measurements in order to determine the radiative capture and/or the fission cross sections of neutron induced reactions on several nuclei $\left({ }^{232} \mathrm{Th}{ }^{233} \mathrm{~Pa},{ }^{233} \mathrm{U}\right)$ which play an important role in the Th-U cycle.
\end{abstract}

\section{Introduction}

Presently nuclear energy provides about $6 \%$ of the total energy consumed in the world and this fraction increases to $16 \%$ if one takes into account only the component of the energy used to generate electricity. At the world level France has a particularly unique position because the electricity production from nuclear origin is extremely high $(\approx 80 \%)$ whereas in the United States for example, although the total amount of electricity provided by nuclear reactors is higher than in France ( 728 TWh compared to $390 \mathrm{TWh}$ ), it only corresponds to $20 \%$ of the total electricity production. Economic experts predict an increase of the world energy consumption of the order of 2 to 3 by 2050. This projected increase in demand will not be easy to satisfy because we know that fossil energies (gas or oil) face a short life before exhaustion of known reserves $(<100$ years) and moreover the use of these fuels have important consequences for our environment (green house effect from emission of $\mathrm{CO}_{2}$ ). Nuclear energy could provide a partial solution to this increased energy demand. However it requires technical solutions that will permit the continuous support of economic growth and improved quality of life together with a minimal impact to human health and environment. An optimum programme for radioactive waste management should include the development of new technologies to reduce the present inventory of nuclear wastes, and simultaneously a more efficient way to use the uranium and thorium natural resources to generate nuclear energy while minimizing waste production over many centuries. If one considers the most commonly used reactor, the pressurized water reactor (PWR) which delivers for example 900 MWe of electricity, it consumes per year of the order of 21.5 tons of enriched uranium (3.5\% of ${ }^{235} \mathrm{U}$ ) obtained from more than 100 tons of natural uranium, which has been extracted from about 70000 tons of ore (depending on the quality of the mine). After irradiation by neutrons and energy production via fission, the spent fuel contains uranium, plutonium, minor actinides (Am, Np, $\mathrm{Cm}$ ) and fission products . The fission products are unavoidable because they correspond to the residues of the energy production via generated heat. About $6.5 \%$ of these are very long lived $\left(\mathrm{T}_{1 / 2}>10^{5}\right.$ years). The other residues are transuraniens (plutonium $200 \mathrm{~kg}$ and minor actinides $16 \mathrm{~kg}$ ) which are produced via single and multiple neutron captures on the fuel.

The future of the nuclear waste management (fission products and minor actinides) is directed in France by a law voted by the parliament on 30 December 1991. The lawmakers decided to establish a 15 year research programme in three directions: 1) Partitioning and transmutation, 2) Geological storage, 3) Waste conditioning and interim storage.

Concerning the first direction, one of the options proposed for transmutation consists in the development of hybrid systems, which couple a high intensity proton accelerator with a sub-critical reactor (cf the contribution of S. Anéfalos at this conference). Another line of study deals with energy production scenarios whose waste stream would, from the start, be orders of magnitude smaller than the present U-Pu cycle. Such scenarios are not necessarily new, but they have been partly overlooked in the past since at that time the waste issue was generally not among the most important concerns of nuclear energy scientists and engineers. In my talk I will focus on the Th-U cycle. This fuel offers a number of advantages. Thorium is more abundant than uranium (factor 3 to 4 ) and in addition monoisotopic $\left({ }^{232} \mathrm{Th}\right)$. The idea to use thorium as fuel to generate electricity goes back to the 60 's, because it is the only nucleus present in nature which can give rise to an excess of fissile material $\left({ }^{233} \mathrm{U}\right)$ in presence of thermal or fast neutrons (breeder reactor), the breeding factor being however not so large (1.0 to 1.15). Last but not least, the actinide waste production with such reactors would be drastically reduced (2 orders of magnitude for a solid fuel with fast neutrons and 3 orders of magnitude for a molten salt liquid fuel and thermal/epithermal neutrons) simply because successive (n, $\gamma$ ) capture reactions on ${ }^{232}$ Th would lead to almost stable isotopes and it requires at least 5 captures to produce the first radiotoxic actinide nucleus $\left({ }^{237} \mathrm{~Np}\right)$.

The use of the U-Pu cycle benefits from about 50 years of research and development. An enormous effort has been 
undertaken concerning experimental nuclear data (crosssections, decay schemes...) but this is not at all the case for the Th-U cycle for which such data are not precise enough or simply missing. In this cycle the fertile nucleus ${ }^{232} \mathrm{Th}$ transforms in a fissile nucleus ${ }^{233} \mathrm{U}$ via a neutron capture and two consecutive $\beta^{-}$decays:

$$
{ }^{232} \mathrm{Th}+\mathrm{n} \rightarrow{ }^{233} \mathrm{Th} \underset{22 \mathrm{~m}}{\stackrel{\beta^{-}}{\rightarrow}} \quad{ }^{233} \mathrm{~Pa} \stackrel{\beta^{-}}{\underset{27 \mathrm{~d}}{\rightarrow}}{ }^{233} \mathrm{U}
$$

One immediately realizes that the nucleus ${ }^{233} \mathrm{~Pa}$ (which corresponds to ${ }^{239} \mathrm{~Np}, \mathrm{~T}_{1 / 2}=2.3$ days, in the usual U-Pu cycle) has a sufficiently long half life $\left(\mathrm{T}_{1 / 2}=27\right.$ days $)$ for capturing neutrons and can therefore seriously perturb the ${ }^{233} \mathrm{U}$ production. The cross sections of interest for this cycle are the following: neutron induced capture reaction ${ }^{232} \mathrm{Th}(\mathrm{n}, \gamma)$ with good accuracy (3\% in particular for the accelerator driven systems), the reactions ${ }^{233} \mathrm{~Pa}(\mathrm{n}, \gamma)$ and ${ }^{233} \mathrm{~Pa}$ (n,fission) for which no experimental data exist and the ${ }^{233} \mathrm{U}$ (n,fission) neutron induced fission reaction for which discrepancies exist between the various databases.

At Bordeaux we have studied over the last two years the ${ }^{232} \mathrm{Th}(\mathrm{n}, \gamma)$ and the ${ }^{233} \mathrm{U}$ (n,fission) reactions with neutrons in the energy range $50 \mathrm{keV}-2 \mathrm{MeV}$. The cross section of the ${ }^{233} \mathrm{~Pa}$ (n,fission) reaction has been determined via a $\left({ }^{3} \mathrm{He}, \mathrm{p}\right)$ transfer reaction at the tandem accelerator of IPN Orsay. The various techniques and the results obtained will be discussed. Our expertise in nuclear data obtained, in the case of reactions induced by neutrons, is strongly linked with the possibilities offered by our old 4MV Van de Graaff accelerator based at the Centre d'Etudes Nuclaires in Bordeaux. Using protons beams of 10-20 $\mu \mathrm{A}$ with energies between 1$3 \mathrm{MeV}$ and either ${ }^{7} \mathrm{Li}$ or ${ }^{3} \mathrm{H}$ targets, it is possible to produce intense monoenergetic neutron beams in the energy range 50 $\mathrm{keV}-2 \mathrm{MeV}$, the experiments being performed with reduced neutron background due to the neutron cave located below the target chamber and the beam line.

\section{Radiative neutron capture of ${ }^{232} \mathrm{Th}$ between $50 \mathrm{keV}$ and $2 \mathrm{MeV}$.}

It is in this energy range that the discrepancies between the various databases are the largest (20-30\%). The current measurement was performed using the activation technique of thorium foils $\left(10 \times 10 \times 0.5 \mathrm{~mm}^{3}\right)$. The irradiation time per sample was of the order of 20 hours. The production rate of ${ }^{233} \mathrm{Th}$ as a function of neutron energy was deduced from delayed $\gamma$-ray spectroscopy of the $312 \mathrm{keV}$ transition deexciting the first excited state of ${ }^{233} \mathrm{U}$, which was populated after two $\beta^{-}$decays $\left(\mathrm{T}_{1 / 2}=27 \mathrm{~d}\right)$.

Below $1 \mathrm{MeV}$, the flux of fast neutrons was determined via activation of gold foils $\left(10 \times 10 \times 0.5 \mathrm{~mm}^{3}\right)$ placed in front and behind the thorium target and protected against possible thermal neutrons by a cadmium box of $1 \mathrm{~mm}$ thickness. The transmutation of ${ }^{197} \mathrm{Au}$ was signed with the $412 \mathrm{keV} \gamma$-ray emitted from the first excited state of ${ }^{198} \mathrm{Hg}$ populated after $\beta^{-}$decay of ${ }^{198} \mathrm{Au}\left(\mathrm{T}_{1 / 2}=2.7 \mathrm{~d}\right)$ and detected out of beam with a $40 \% \mathrm{Ge}$ detector. Above $1 \mathrm{MeV}$ neutron energy, the flux was furthermore determined by measuring the fission rate of a thin ${ }^{235} \mathrm{U}$ target $\left(50 \mu \mathrm{g} / \mathrm{cm}^{2}\right)$ with solar cell detectors. For those two samples, the cross sections ${ }^{197} \mathrm{Au}(\mathrm{n}, \gamma)$ and ${ }^{235} \mathrm{U}$ (n,fission) are known to better than $4 \%$ and therefore provide excellent probes to accurately monitor the neutron flux.

The cross section values obtained for 22 neutron energies are presented in Fig. 1 and they are in fair agreement with the Japanese database JENDL3 [1].

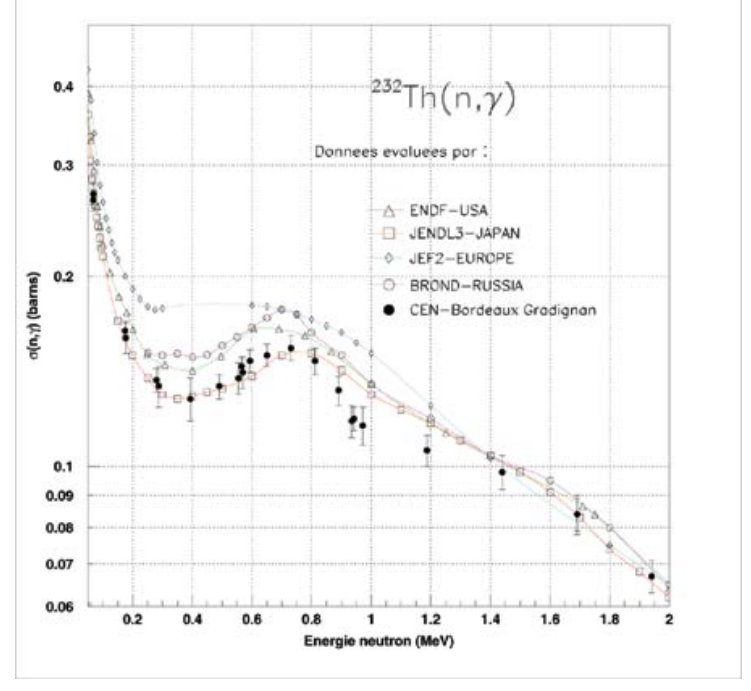

Figure 1.

\section{Determination of the ${ }^{233} \mathrm{~Pa}(\mathrm{n}$,fission) cross section between 0.5 and 10 MeV.}

The extremely high activity $\left(\approx 10^{9} \mathrm{~Bq} / \mu \mathrm{g}\right)$ of ${ }^{233} \mathrm{~Pa}$ and its short half life $(27 \mathrm{~d})$ are a challenge for experimentalists. To overcome these constraints, we have decided to form the fissionning nucleus ${ }^{234} \mathrm{~Pa}$ via the ${ }^{232} \mathrm{Th}\left({ }^{3} \mathrm{He}, \mathrm{p}\right)$ transfer reaction. By detecting the emitted protons in coincidence with the fission fragments, it is possible to deduce the fission probability of the nucleus ${ }^{234} \mathrm{~Pa}$ as a function of its excitation energy. Then, the cross section for the neutron induced fission reaction ${ }^{233} \mathrm{~Pa}(\mathrm{n}$,fission) is determined by the product of this fission probability times the cross section for formation of the compound nucleus ${ }^{234} \mathrm{~Pa}$ for a given neutron incident energy (and therefore for given excitation energy of ${ }^{234} \mathrm{~Pa}$ ). This cross section was calculated within the framework of the optical model by the group J.P. Delaroche et al. from the Bruyères le Châtel laboratory. The experiment was performed at the IPN Orsay tandem accelerator with ${ }^{3} \mathrm{He}$ beams at three bombarding energies 24, 27 and 30 $\mathrm{MeV}$ and using ${ }^{232} \mathrm{Th}$ targets of $100 \mu \mathrm{g} / \mathrm{cm}^{2}$ deposited on a carbon backing of $50 \mu \mathrm{g} / \mathrm{cm}^{2}$. The various reaction channels $\left({ }^{3} \mathrm{He}, \mathrm{p}\right){ }^{234} \mathrm{~Pa},\left({ }^{3} \mathrm{He}, \mathrm{d}\right){ }^{233} \mathrm{~Pa},\left({ }^{3} \mathrm{He}, \mathrm{t}\right){ }^{232} \mathrm{~Pa}$ and $\left.\left({ }^{3} \mathrm{He}, \alpha\right)\right)^{231} \mathrm{Th}$ 

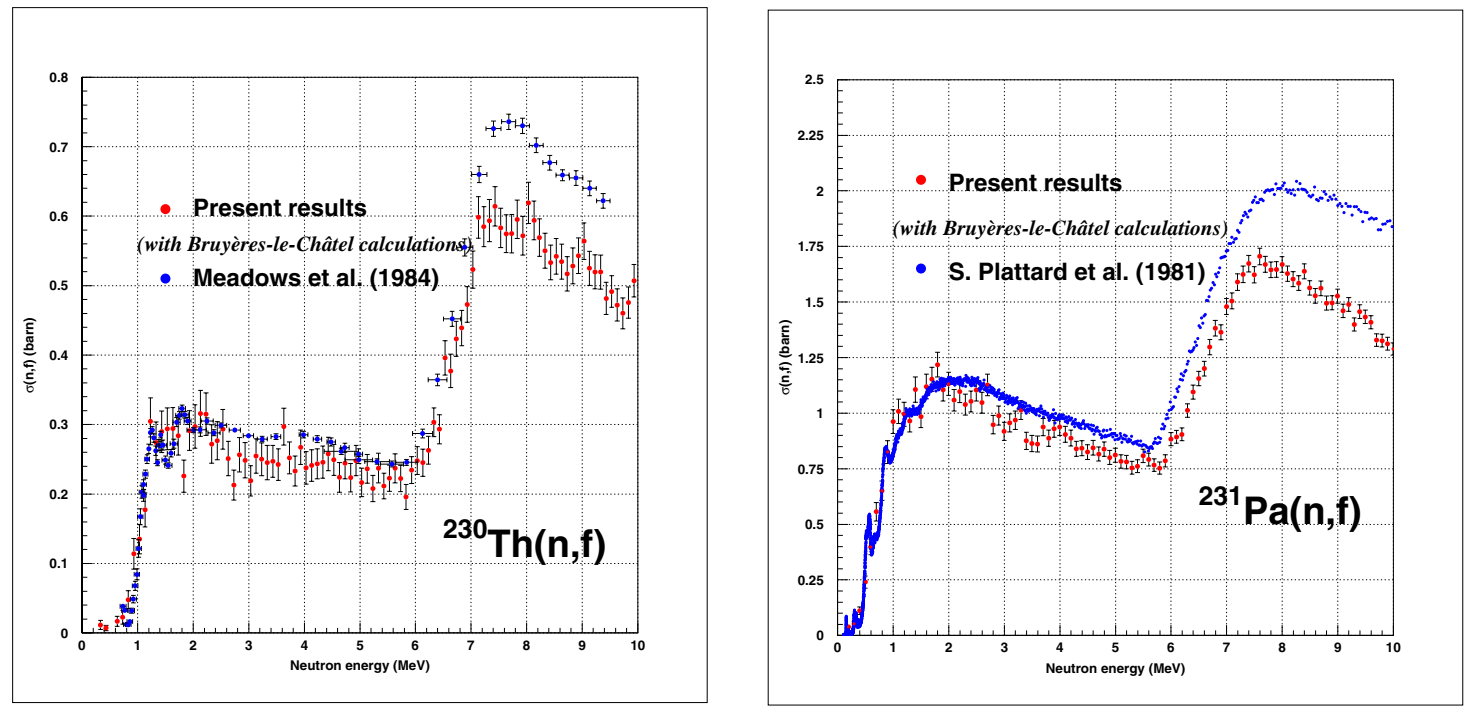

Figure 2.

were selected by detection of the light ejectile $\mathrm{p}, \mathrm{d}$, t or $\alpha$ with two $\Delta \mathrm{E}$-E telescopes placed at $5 \mathrm{~cm}$ from the target and at $90^{\circ}$ and $130^{\circ}$ with respect to the beam axis. Fission fragments were detected with a set of fifteen solar cells placed at $5 \mathrm{~cm}$ from the target between $27^{\circ}$ and $210^{\circ}$ with respect to the beam. The fission probability of the nucleus ${ }^{234} \mathrm{~Pa}\left({ }^{233} \mathrm{~Pa}, \ldots\right)$ was deduced from the ratio of the number of corresponding charged particles detected in coincidence with fission fragments and the number of charged particles detected in singles after correction for the solid angle of the solar cells. The determination of the fission cross section, as explained previously, assumes that the distribution of states populated in the transfer reaction and the neutron induced reaction are very similar (similar quantum numbers). We have tested this hypothesis by comparing the results obtained using the ${ }^{232} \mathrm{Th}\left({ }^{3} \mathrm{He}, \mathrm{t}\right){ }^{232} \mathrm{~Pa}$ and ${ }^{232} \mathrm{Th}\left({ }^{3} \mathrm{He}, \alpha\right){ }^{231} \mathrm{Th}$ channels with direct measurements of the neutron induced ${ }^{231} \mathrm{~Pa}$ (n,fission) and ${ }^{230} \mathrm{Th}$ (n,fission) reactions. As shown in Fig. 2 our values are in fair agreement with the direct measurements at least up to $6 \mathrm{MeV}$ and therefore validate our present applied procedure. The results for the fissionning nucleus ${ }^{234} \mathrm{~Pa}$ are presented in Fig. 3. The onset of fission at $\approx 1 \mathrm{MeV}$ neutron energy is in good agreement with the American ENDF data base whereas at higher neutron energies up to $6 \mathrm{MeV}$ our results confirm the predictions of the Japanese data base.

\section{Cross section for the ${ }^{233} \mathbf{U}$ (n,fission) reaction between $50 \mathrm{keV}$ and 2 MeV.}

The dispersion of the experimental fission cross sections in this neutron energy range is of the order of $20-30 \%$. The precision required for the Th- $\mathrm{U}$ cycle is $\approx 5 \%$. We have undertaken a series of measurements at the CENBG (in collaboration with the group of ISN Grenoble) using the experimental set up presented schematically in Fig. 4. Knowing the number $\mathrm{N}_{f}$ of detected fission events, the neutron flux $\Phi$ deduced either from the gold foil activation and/or the fission rate of ${ }^{235} \mathrm{U}$, the number $\mathrm{N}$ of target nuclei determined by counting with a silicon detector the $\alpha$ decays of ${ }^{233} \mathrm{U}$, it is then easy to calculate the cross section for the reaction ${ }^{233} \mathrm{U}+\mathrm{n}$ as a function of neutron energy. This programme has been started recently and experiments are under analysis.

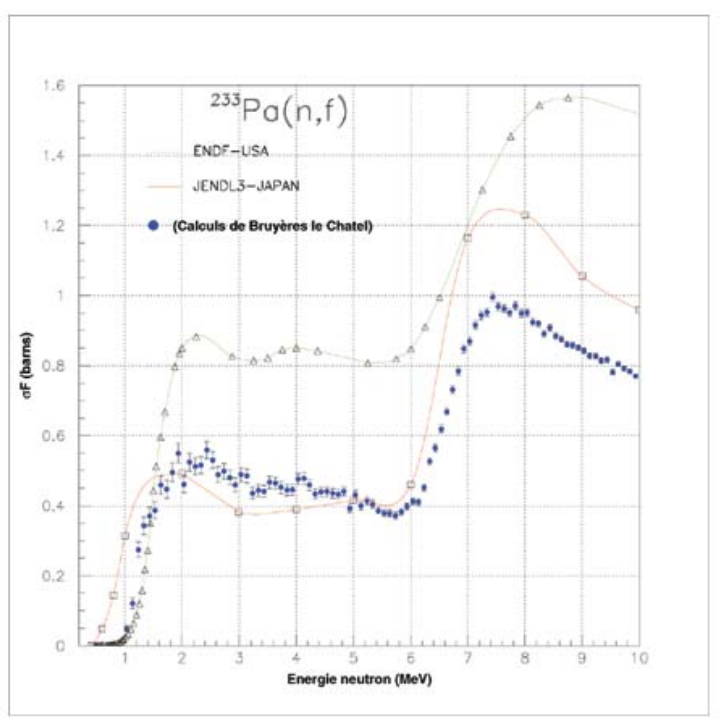

Figure 3. 


\section{Conclusion}

The different experiments presented in this talk require a variety of techniques very often used by nuclear physicists involved in fundamental research programmes. Hopefully I have convinced you that these techniques can also be used for more applied work such as studies of new fuel cycles. In the near future and in the frame of the nuclear waste programme, we plan to study the ${ }^{242,244} \mathrm{Cm}$ (n,fission) reactions using the transfer reactions ${ }^{241,243} \mathrm{Am}\left({ }^{3} \mathrm{He}, \mathrm{p}\right)$ and applying the same method as used for ${ }^{233} \mathrm{~Pa}$. A new in beam detection system for photons $\left(\mathrm{C}_{6} \mathrm{D}_{6}\right.$ liquid scintillator), which can be used either with neutron or charged particle beams, has also been developed and we envisage to use this new device in order to determine the cross section of the capture reaction ${ }^{233} \mathrm{~Pa}(\mathrm{n}, \gamma)$ which is of interest for the Th- $\mathrm{U}$ fuel cycle.

\section{Acknowledgments}

I would like to thank all my collaborators at the CENBG (M. Aiche, G. Barreau, S. Boyer, S. Czajkowski, D. Dassie, C. Grosjean, A. Guiral, D. Karamanis, M. Petit and J. Wilson) as well as those from IPN Orsay (S. Fortier), ISN Grenoble (A Billebaud, R. Brissot M. Kerveno, C. Le Brun and L. Perrot) and CEA/Saclay (E. Bouchez, F. Gunsing, A.Hurstel, Y. Le Coz, R. Lucas, M. Rejmund and C. Theisen). This work was partly supported by the CNRS programme PACE (Programme Aval du Cycle Electronucléaires) as well as by the Conseil Régional d'Aquitaine.

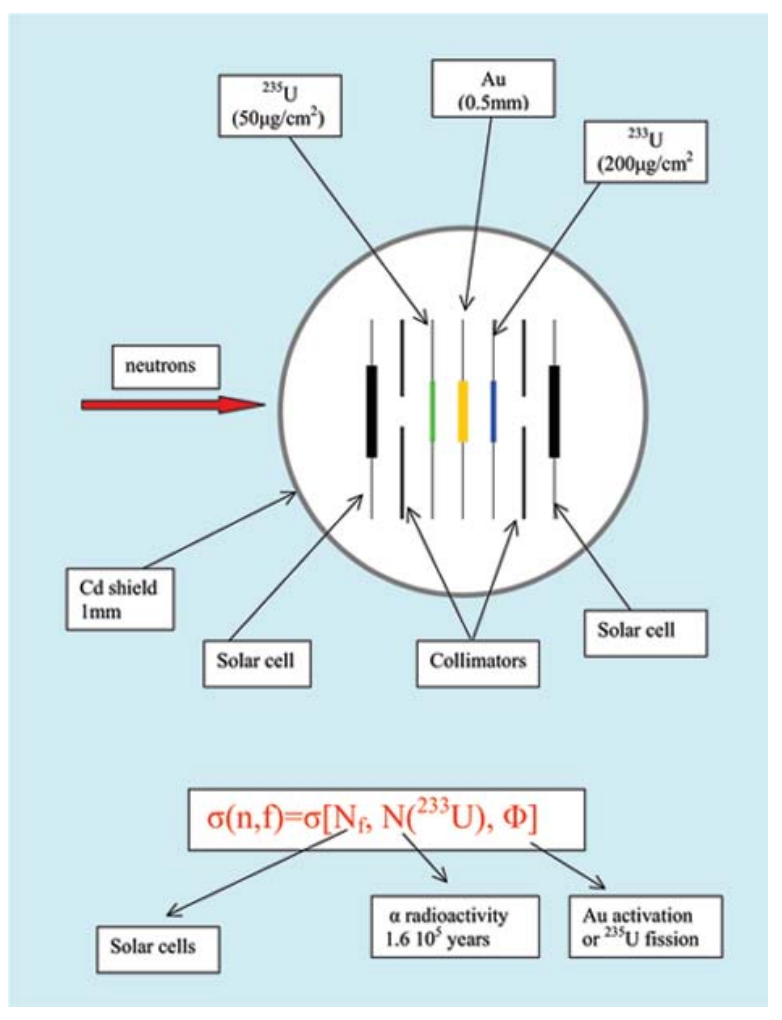

Figure 4.

\section{References}

[1] D. Karamanis et al. Nucl. Sci. and Eng. 139, 282 (2001). 